\title{
Planck 2013 results. XXVII. Doppler boosting of the CMB: Eppur si muove ${ }^{\star}$
}

Planck Collaboration: N. Aghanim ${ }^{56}$, C. Armitage-Caplan ${ }^{85}$, M. Arnaud ${ }^{69}$, M. Ashdown ${ }^{66,6}$, F. Atrio-Barandela ${ }^{16}$, J. Aumont ${ }^{56}$, C. Baccigalupi $^{79}$, A. J. Banday ${ }^{87,9}$, R. B. Barreiro ${ }^{63}$, J. G. Bartlett ${ }^{1,64}$, K. Benabed ${ }^{57,86}$, A. Benoit-Lévy ${ }^{22,57,86}$, J.-P. Bernard ${ }^{87,9}$, M. Bersanelli ${ }^{34,47}$, P. Bielewicz ${ }^{87,9,79}$, J. Bobin ${ }^{69}$, J. J. Bock ${ }^{64,10}$, J. R. Bond ${ }^{8}$, J. Borrill ${ }^{12,83}$, F. R. Bouchet ${ }^{57,86}$, M. Bridges ${ }^{66,6,60}$, C. Burigana ${ }^{46,32}$, R. C. Butler ${ }^{46}$, J.-F. Cardoso ${ }^{70,1,57}$, A. Catalano ${ }^{71,68}$, A. Challinor ${ }^{60,66,11}$, A. Chamballu ${ }^{69,13,56}$, H. C. Chiang ${ }^{26,7}$, L.-Y Chiang ${ }^{59}$, P. R. Christensen ${ }^{76,37}$, D. L. Clements ${ }^{54}$,

L. P. L. Colombo ${ }^{21,64}$, F. Couchot ${ }^{67}$, B. P. Crill ${ }^{64,77}$, A. Curto ${ }^{6,63}$, F. Cuttaia ${ }^{46}$, L. Danese ${ }^{79}$, R. D. Davies ${ }^{65}$, R. J. Davis ${ }^{65}$, P. de Bernardis ${ }^{33}$,

A. de Rosa ${ }^{46}$, G. de Zotti ${ }^{42,79}$, J. Delabrouille ${ }^{1}$, J. M. Diego ${ }^{63}$, S. Donzellii7 ${ }^{47}$, O. Doré ${ }^{64,10}$, X. Dupac ${ }^{39}$, G. Efstathiou ${ }^{60}$, T. A. Enßlinn ${ }^{74}$, H. K. Eriksen ${ }^{61}$, F. Finelli ${ }^{46,48}$, O. Forni ${ }^{87,9}$, M. Frailis ${ }^{44}$, E. Franceschi ${ }^{46}$, S. Galeotta ${ }^{44}$, K. Ganga ${ }^{1}$, M. Giard ${ }^{87,9}$, G. Giardino $^{40}$,

J. González-Nuevo ${ }^{63,79}$, K. M. Górski ${ }^{64,89}$, S. Gratton ${ }^{66,60}$, A. Gregorio ${ }^{35,44,50}$, A. Gruppuso ${ }^{46}$, F. K. Hansen ${ }^{61}$, D. Hanson ${ }^{75,64,8}$, D. L. Harrison ${ }^{60,66}$, G. Helou ${ }^{10}$, S. R. Hildebrandt ${ }^{10}$, E. Hivon ${ }^{57,86}$, M. Hobson ${ }^{6}$, W. A. Holmes ${ }^{64}$, W. Hovest ${ }^{74}$, K. M. Huffenberger ${ }^{24}$, W. C. Jones ${ }^{26}$, M. Juvela ${ }^{25}$,

E. Keihänen ${ }^{25}$, R. Keskitalo ${ }^{19,12}$, T. S. Kisner ${ }^{73}$, J. Knoche ${ }^{74}$, L. Knox ${ }^{28}$, M. Kunz ${ }^{15,56,3}$, H. Kurki-Suonio ${ }^{25,41}$, A. Lähteenmäki ${ }^{2,41}$,

J.-M. Lamarre ${ }^{68}$, A. Lasenby ${ }^{6,66}$, R. J. Laureijs ${ }^{40}$, C. R. Lawrence ${ }^{64}$, R. Leonardi ${ }^{39}$, A. Lewis ${ }^{23}$, M. Liguori ${ }^{31}$, P. B. Lilje ${ }^{61}$, M. Linden-Vørnle ${ }^{14}$, M. López-Caniego ${ }^{63}$, P. M. Lubin ${ }^{29}$, J. F. Macías-Pérez ${ }^{71}$, N. Mandolesi ${ }^{46,5,32}$, M. Maris ${ }^{44}$, D. J. Marshall ${ }^{69}$, P. G. Martin ${ }^{8}$,

E. Martínez-González ${ }^{63}$, S. Masi ${ }^{33}$, M. Massardi ${ }^{45}$, S. Matarrese ${ }^{31}$, P. Mazzotta ${ }^{36}$, P. R. Meinhold ${ }^{29}$, A. Melchiorri ${ }^{33,49}$, L. Mendes $^{39}$, M. Migliaccio ${ }^{60,66}$, S. Mitra ${ }^{53,64}$, A. Moneti ${ }^{57}$, L. Montier ${ }^{87,9}$, G. Morgante ${ }^{46}$, D. Mortlock ${ }^{54}$, A. Moss ${ }^{81}$, D. Munshi ${ }^{80}$, P. Naselsky ${ }^{76,37}$, F. Nati ${ }^{33}$, P. Natoli ${ }^{32,4,46}$, H. U. Nørgaard-Nielsen ${ }^{14}$, F. Noviello ${ }^{65}$, D. Novikov ${ }^{54}$, I. Novikov ${ }^{76}$, S. Osborne ${ }^{84}$, C. A. Oxborrow ${ }^{14}$, L. Pagano ${ }^{33,49}$, F. Pajot ${ }^{56}$, D. Paoletti ${ }^{46,48}$, F. Pasian ${ }^{44}$, G. Patanchon ${ }^{1}$, O. Perdereau ${ }^{67}$, F. Perrotta ${ }^{79}$, F. Piacentinii ${ }^{33}$, E. Pierpaoli ${ }^{21}$, D. Pietrobon ${ }^{64}$, S. Plaszczynski ${ }^{67}$, E. Pointecouteau ${ }^{87,9}$, G. Polenta ${ }^{4,43}$, N. Ponthieu ${ }^{56,51}$, L. Popa ${ }^{58}$, G. W. Pratt ${ }^{69}$, G. Prézeau ${ }^{10,64}$, J.-L. Puget ${ }^{56}$, J. P. Rachen ${ }^{18,74}$, W. T. Reach ${ }^{88}$, M. Reinecke ${ }^{74}$, S. Ricciardi ${ }^{46}$, T. Riller ${ }^{74}$, I. Ristorcelli ${ }^{87,9}$, G. Rocha ${ }^{64,10}$, C. Rosset ${ }^{1}$, J. A. Rubiño-Martín ${ }^{62,38}$, B. Rusholme ${ }^{55}$, D. Santos ${ }^{71}$,

G. Savini ${ }^{78}$, D. Scott ${ }^{20, \star \star}$, M. D. Seiffert ${ }^{64,10}$, E. P. S. Shellard ${ }^{11}$, L. D. Spencer ${ }^{80}$, R. Sunyaev ${ }^{74,82}$, F. Sureau ${ }^{69}$, A.-S. Suur-Uski $^{25,41}$,

J.-F. Sygnet ${ }^{57}$, J. A. Tauber ${ }^{40}$, D. Tavagnacco ${ }^{44,35}$, L. Terenzi ${ }^{46}$, L. Toffolatti ${ }^{17,63}$, M. Tomasi $^{34,47}$, M. Tristram ${ }^{67}$, M. Tucci $^{15,67}$, M. Türler $^{52}$, L. Valenziano ${ }^{46}$, J. Valiviita ${ }^{41,25,61}$, B. Van Tent ${ }^{72}$, P. Vielva ${ }^{63}$, F. Villa ${ }^{46}$, N. Vittorio ${ }^{36}$, L. A. Wade ${ }^{64}$, B. D. Wandelt ${ }^{57,86,30}$, M. White $^{27}$, D. Yvon ${ }^{13}$, A. Zacchei ${ }^{44}$, J. P. Zibin ${ }^{20}$, and A. Zonca ${ }^{29}$

(Affiliations can be found after the references)

Received 23 March 2013 / Accepted 3 January 2014

\section{ABSTRACT}

Our velocity relative to the rest frame of the cosmic microwave background (CMB) generates a dipole temperature anisotropy on the sky which has been well measured for more than 30 years, and has an accepted amplitude of $v / c=1.23 \times 10^{-3}$, or $v=369 \mathrm{~km} \mathrm{~s}^{-1}$. In addition to this signal generated by Doppler boosting of the CMB monopole, our motion also modulates and aberrates the CMB temperature fluctuations (as well as every other source of radiation at cosmological distances). This is an order $10^{-3}$ effect applied to fluctuations which are already one part in roughly $10^{5}$, so it is quite small. Nevertheless, it becomes detectable with the all-sky coverage, high angular resolution, and low noise levels of the Planck satellite. Here we report a first measurement of this velocity signature using the aberration and modulation effects on the CMB temperature anisotropies, finding a component in the known dipole direction, $(l, b)=\left(264^{\circ}, 48^{\circ}\right)$, of $384 \mathrm{~km} \mathrm{~s}^{-1} \pm 78 \mathrm{~km} \mathrm{~s}^{-1}$ (stat.) $\pm 115 \mathrm{~km} \mathrm{~s}^{-1}$ (syst.). This is a significant confirmation of the expected velocity.

Key words. cosmology: observations - cosmic background radiation - reference systems - relativistic processes

\section{Introduction}

This paper, one of a set associated with the 2013 release of data from the Planck ${ }^{1}$ mission (Planck Collaboration I 2014),

\footnotetext{
* "And yet it moves", the phrase popularly attributed to Galileo Galilei after being forced to recant his view that the Earth goes around the Sun.

$\star \star$ Corresponding author: Douglas Scott,

e-mail: dscott@phas.ubc.ca

1 Planck (http://www.esa.int/Planck) is a project of the European Space Agency (ESA) with instruments provided by two scientific consortia funded by ESA member states (in particular the lead countries France and Italy), with contributions from NASA (USA) and telescope reflectors provided by a collaboration between ESA and a scientific consortium led and funded by Denmark.
}

presents a study of Doppler boosting effects using the smallscale temperature fluctuations of the Planck cosmic microwave background (CMB) maps.

Observations of the relatively large amplitude CMB temperature dipole are usually taken to indicate that our Solar System barycentre is in motion with respect to the CMB frame (defined precisely below). Assuming that the observed temperature dipole is due entirely to Doppler boosting of the CMB monopole, one infers a velocity $v=(369 \pm 0.9) \mathrm{km} \mathrm{s}^{-1}$ in the direction $(l, b)=\left(263.99 \pm 0.14,48^{\circ} .26 \pm 0.03\right)$, on the boundary of the constellations of Crater and Leo (Kogut et al. 1993; Fixsen et al. 1996; Hinshaw et al. 2009).

In addition to Doppler boosting of the CMB monopole, velocity effects also boost the order $10^{-5}$ primordial temperature 
fluctuations. There are two observable effects here, both at a level of $\beta \equiv v / c=1.23 \times 10^{-3}$. First, there is a Doppler "modulation" effect, which amplifies the apparent temperature fluctuations in the velocity direction, and reduces them in the opposite direction. This is the same effect which converts a portion of the CMB monopole into the observed dipole. The effect on the CMB fluctuations is to increase the amplitude of the power spectrum by approximately $0.25 \%$ in the velocity direction, and decrease it correspondingly in the anti-direction. Second, there is an "aberration" effect, in which the apparent arrival direction of CMB photons is pushed towards the velocity direction. This effect is small, but non-negligible. The expected velocity induces a peak deflection of $\beta=4.2$ and a root-mean-squared (rms) deflection over the sky of $3^{\prime}$, comparable to the effects of gravitational lensing by large-scale structure, which are discussed in Planck Collaboration XVII (2014). The aberration effect squashes the anisotropy pattern on one side of the sky and stretches it on the other, effectively changing the angular scale. Close to the velocity direction we expect that the power spectrum of the temperature anisotropies, $C_{\ell}$, will be shifted so that, e.g., $\ell=1000 \rightarrow \ell=1001$, while $\ell=1000 \rightarrow \ell=999$ in the antidirection. In Fig. 1 we plot an exaggerated illustration of the aberration and modulation effects. For completeness we should point out that there is a third effect, a quadrupole of amplitude $\beta^{2}$ induced by the dipole (see Kamionkowski \& Knox 2003). However, extracting this signal would require extreme levels of precision for foreground modelling at the quadrupole scale, and we do not discuss it further.

In this paper, we will present a measurement of $\beta$, exploiting the distinctive statistical signatures of the aberration and modulation effects on the high- $\ell$ CMB temperature anisotropies. In addition to our interest in making an independent measurement of the velocity signature, the effects which velocity generates on the CMB fluctuations provide a source of potential bias or confusion for several aspects of the Planck data. In particular, velocity effects couple to measurements of: primordial " $\tau_{\mathrm{NL}}$ "-type non-Gaussianity, as discussed in Planck Collaboration XXIV (2014); statistical anisotropy of the primordial CMB fluctuations, as discussed in Planck Collaboration XXIII (2014); and gravitational lensing, as discussed in Planck Collaboration XVII (2014). There are also aspects of the Planck analysis for which velocity effects are believed to be negligible, but only if they are present at the expected level. One example is measurement of $f_{\mathrm{NL}}$-type non-Gaussianity, as discussed in Catena et al. (2013). Another example is power spectrum estimation - as discussed above, velocity effects change the angular scale of the acoustic peaks in the CMB power spectrum. Averaged over the full sky this effect is strongly suppressed, as the expansion and contraction of scales on opposing hemispheres cancel out. However the application of a sky mask breaks this cancellation to some extent, and can potentially be important for parameter estimation (Pereira et al. 2010; Catena \& Notari 2013). For the 143 and $217 \mathrm{GHz}$ analysis mask used in the fiducial Planck CMB likelihood (Planck Collaboration XV 2014), the average lensing convergence field associated with the aberration effect (on the portion of the sky which is unmasked) has a value which is $13 \%$ of its peak value, corresponding to an expected average lensing convergence of $\beta \times 0.13=1.5 \times 10^{-4}$. This will shift the angular scale of the acoustic peaks by the same fraction, which is degenerate with a change in the angular size of the sound horizon at last scattering, $\theta_{*}$ (Burles \& Rappaport 2006). A $1.5 \times 10^{-4}$ shift in $\theta_{*}$ is just under $25 \%$ of the Planck uncertainty on this parameter, as reported in Planck Collaboration XVI (2014) small enough to be neglected, though not dramatically so. This
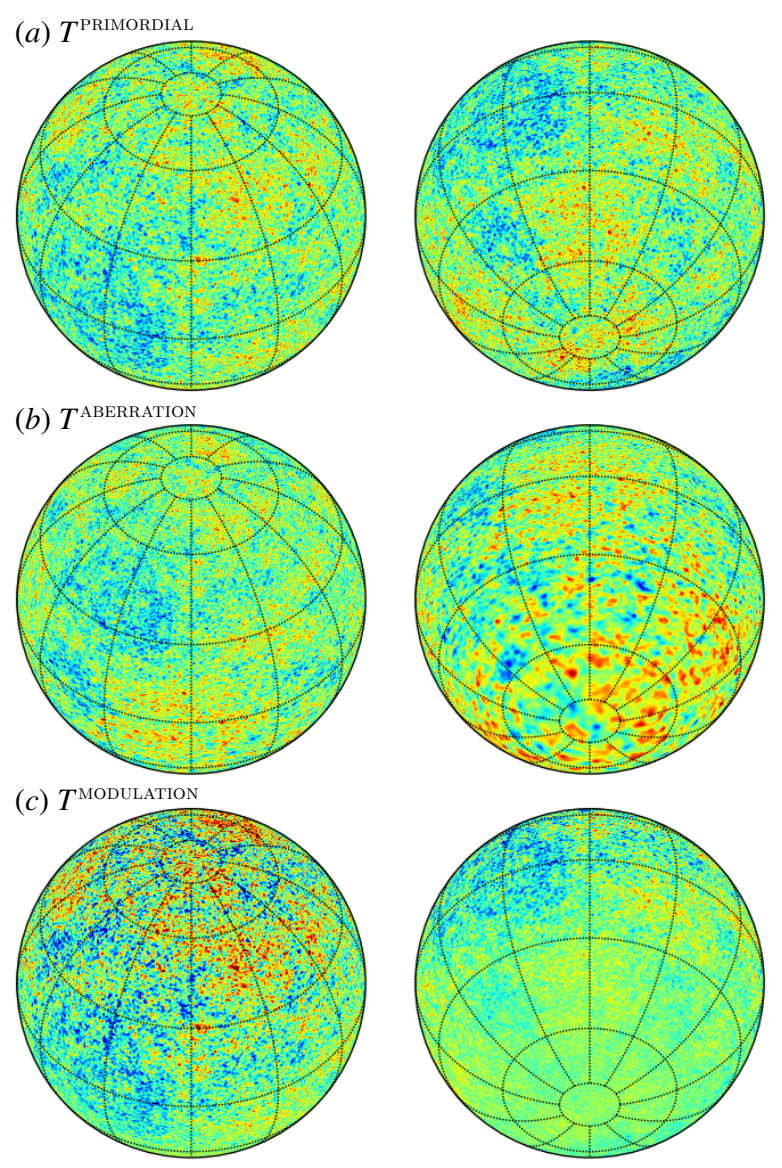

Fig. 1. Exaggerated illustration of the aberration and Doppler modulation effects, in orthographic projection, for a velocity $v=$ $260000 \mathrm{~km} \mathrm{~s}^{-1}=0.85 c$ (approximately 700 times larger than the expected magnitude) towards the northern pole (indicated by meridians in the upper half of each image on the left). The aberration component of the effect shifts the apparent position of fluctuations towards the velocity direction, while the modulation component enhances the fluctuations in the velocity direction and suppresses them in the anti-velocity direction.

therefore motivates us to test that the observed aberration signal is not significantly larger than expected. With such a confirmation in hand, a logical next step is to correct for these effects by a process of de-boosting the observed temperature (Notari \& Quartin 2012; Yoho et al. 2012). Indeed, an analysis of maps corrected for the modulation effect described here is performed in Planck Collaboration XXIII (2014).

Before proceeding to discuss the aberration and modulation effects in more detail, we note that in addition to the overall peculiar velocity of our Solar System with respect to the CMB, there is an additional time-dependent velocity effect from the orbit of Planck (at L2, along with the Earth) about the Sun. This velocity has an average amplitude of approximately $30 \mathrm{~km} \mathrm{~s}^{-1}$, less than one-tenth the size of the primary velocity effect. The aberration component of the orbital velocity (more commonly referred to in astronomy as "stellar aberration") has a maximum amplitude of 20.5 and is corrected for in the satellite pointing. The modulation effect for the orbital velocity switches signs between each 6-month survey, and so is suppressed when using multiple surveys to make maps (as we do here, with the nominal 
Planck maps, based on a little more than two surveys), and so we will not consider it further ${ }^{2}$.

\section{Aberration and modulation}

Here we will present a more quantitative description of the aberration and modulation effects described above. To begin, note that, by construction, the peculiar velocity, $\boldsymbol{\beta}$, measures the velocity of our Solar System barycentre relative to a frame, called the CMB frame, in which the temperature dipole, $a_{1 m}$, vanishes. However, in completely subtracting the dipole, this frame would not coincide with a suitably-defined average $\mathrm{CMB}$ frame, in which an observer would expect to see a dipole $C_{1} \sim 10^{-10}$, given by the Sachs-Wolfe and integrated Sachs-Wolfe effects (see Zibin \& Scott 2008 for discussion of cosmic variance in the CMB monopole and dipole). The velocity difference between these two frames is, however, small, at the level of $1 \%$ of our observed $v$.

If $T^{\prime}$ and $\hat{\boldsymbol{n}}^{\prime}$ are the CMB temperature and direction as viewed in the CMB frame, then the temperature in the observed frame is given by the Lorentz transformation (see, e.g., Challinor \& van Leeuwen 2002; Sollom 2010),

$T(\hat{\boldsymbol{n}})=\frac{T^{\prime}\left(\hat{\boldsymbol{n}}^{\prime}\right)}{\gamma(1-\hat{\boldsymbol{n}} \cdot \boldsymbol{\beta})}$,

where the observed direction $\hat{\boldsymbol{n}}$ is given by

$\hat{\boldsymbol{n}}=\frac{\hat{\boldsymbol{n}}^{\prime}+\left[(\gamma-1) \hat{\boldsymbol{n}}^{\prime} \cdot \hat{\boldsymbol{v}}+\gamma \beta\right] \hat{\boldsymbol{v}}}{\gamma\left(1+\hat{\boldsymbol{n}}^{\prime} \cdot \boldsymbol{\beta}\right)}$,

and $\gamma \equiv\left(1-\beta^{2}\right)^{-1 / 2}$. Expanding to linear order in $\beta$ gives

$T^{\prime}\left(\hat{\boldsymbol{n}}^{\prime}\right)=T^{\prime}(\hat{\boldsymbol{n}}-\nabla(\hat{\boldsymbol{n}} \cdot \boldsymbol{\beta})) \equiv T_{0}+\delta T^{\prime}(\hat{\boldsymbol{n}}-\nabla(\hat{\boldsymbol{n}} \cdot \boldsymbol{\beta}))$,

so that we can write the observed temperature fluctuations as

$\delta T(\hat{\boldsymbol{n}})=T_{0} \hat{\boldsymbol{n}} \cdot \boldsymbol{\beta}+\delta T^{\prime}(\hat{\boldsymbol{n}}-\nabla(\hat{\boldsymbol{n}} \cdot \boldsymbol{\beta}))(1+\hat{\boldsymbol{n}} \cdot \boldsymbol{\beta})$.

Here $T_{0}=(2.7255 \pm 0.0006) \mathrm{K}$ is the CMB mean temperature (Fixsen 2009). The first term on the right-hand side of Eq. (4) is the temperature dipole. The remaining term represents the fluctuations, aberrated by deflection $\nabla(\hat{\boldsymbol{n}} \cdot \boldsymbol{\beta})$ and modulated by the factor $(1+\hat{\boldsymbol{n}} \cdot \boldsymbol{\beta})$.

The Planck detectors can be modelled as measuring differential changes in the CMB intensity at frequency $v$ given by

$I_{v}(v, \hat{\boldsymbol{n}})=\frac{2 h v^{3}}{c^{2}} \frac{1}{\exp \left[h v / k_{\mathrm{B}} T(\hat{\boldsymbol{n}})\right]-1}$.

We can expand the measured intensity difference according to

$\delta I_{v}(v, \hat{\boldsymbol{n}})=\left.\frac{\mathrm{d} I_{v}}{\mathrm{~d} T}\right|_{T_{0}} \delta T(\hat{\boldsymbol{n}})+\left.\frac{1}{2} \frac{\mathrm{d}^{2} I_{v}}{\mathrm{~d} T^{2}}\right|_{T_{0}} \delta T^{2}(\hat{\boldsymbol{n}})+\ldots$

Substituting Eq. (4) and dropping terms of order $\beta^{2}$ and $\left(\delta T^{\prime}\right)^{2}$, we find

$\delta I_{v}(v, \hat{\boldsymbol{n}})=\left.\frac{\mathrm{d} I_{v}}{\mathrm{~d} T}\right|_{T_{0}}\left[T_{0} \hat{\boldsymbol{n}} \cdot \boldsymbol{\beta}+\delta T^{\prime}\left(\hat{\boldsymbol{n}}^{\prime}\right)\left(1+b_{v} \hat{\boldsymbol{n}} \cdot \boldsymbol{\beta}\right)\right]$,

\footnotetext{
2 Note that in both stellar and cosmological cases, the aberration is the result of local velocity differences (Eisner 1967; Phipps 1989): in the former case, between Earth's velocity at different times of the year, and in the latter between the actual and CMB frames.
}

where the frequency dependent boost factor $b_{v}$ is given by

$b_{v}=\frac{v}{v_{0}} \operatorname{coth}\left(\frac{v}{2 v_{0}}\right)-1$

with $v_{0} \equiv k_{\mathrm{B}} T_{0} / h \simeq 57 \mathrm{GHz}$. Integrated over the Planck bandpasses for the 143 and $217 \mathrm{GHz}$ channels, these effective boost factors are given by $b_{143}=1.961 \pm 0.015$, and $b_{217}=$ $3.071 \pm 0.018$, where the uncertainties represent the scatter between the individual detector bandpasses at each frequency. We will approximate these boost factors simply as $b_{143}=2$ and $b_{217}=3$, which is sufficiently accurate for the precision of our measurement.

In the mapmaking process, the fluctuations are assumed to satisfy only the linear term in Eq. (6). Therefore, the inferred temperature fluctuations will be

$\frac{\delta I_{v}(v, \hat{\boldsymbol{n}})}{\mathrm{d} I_{v} /\left.\mathrm{d} T\right|_{T_{0}}}=T_{0} \hat{\boldsymbol{n}} \cdot \boldsymbol{\beta}+\delta T^{\prime}(\hat{\boldsymbol{n}}-\nabla(\hat{\boldsymbol{n}} \cdot \boldsymbol{\beta}))\left(1+b_{v} \hat{\boldsymbol{n}} \cdot \boldsymbol{\beta}\right)$.

Notice that, compared with the actual fluctuations in Eq. (4), the modulation term in Eq. (9) has taken on a peculiar frequency dependence, represented by $b_{v}$. This has arisen due to the coupling between the fluctuations and the dipole, $T_{0} \hat{\boldsymbol{n}} \cdot \boldsymbol{\beta}$, which leads to a second-order term in the expansion of Eq. (6). Intuitively, the CMB temperature varies from one side of the sky to the other at the $3 \mathrm{mK}$ level. Therefore so does the calibration factor $\mathrm{d} I_{v} / \mathrm{d} T$, as represented by the second derivative $\mathrm{d}^{2} I_{v} / \mathrm{d} T^{2}$. We note that such a frequency-dependent modulation is not uniquely a velocity effect, but would have arisen in the presence of any sufficiently large temperature fluctuation. Of course if we measured $T(\hat{\boldsymbol{n}})$ directly (for example by measuring $I_{v}(v, \hat{\boldsymbol{n}})$ at a large number of frequencies), we would measure the true fluctuations, Eq. (4), i.e., we would have a boost factor of $b_{v}=1$. However, this is not what happens in practice, and hence the velocitydriven modulation has a spectrum which mixes in a $\mathrm{d}^{2} I_{v} / \mathrm{d} T^{2}$ dependence.

\section{Methodology}

The statistical properties of the aberration-induced stretching and compression of the CMB anisotropies are manifest in "statistically anisotropic" contributions to the covariance matrix of the CMB, which we can use to reconstruct the velocity vector (Burles \& Rappaport 2006; Kosowsky \& Kahniashvili 2011; Amendola et al. 2011). To discuss these it will be useful to introduce the harmonic transform of the peculiar velocity vector, given by

$\beta_{L M}=\int \mathrm{d} \hat{\boldsymbol{n}} Y_{L M}^{*}(\hat{\boldsymbol{n}}) \boldsymbol{\beta} \cdot \hat{\boldsymbol{n}}$.

Here $\beta_{L M}$ is only non-zero for dipole modes (with $L=1$ ). Although most of our equations will be written in terms of $\beta_{L M}$, for simplicity of interpretation we will present results in a specific choice of basis for the three dipole modes of orthonormal unit vectors, labelled $\boldsymbol{\beta}_{\|}$(along the expected velocity direction), $\boldsymbol{\beta}_{\times}$(perpendicular to $\boldsymbol{\beta}_{\|}$and parallel to the Galactic plane, near its centre), and the remaining perpendicular mode $\beta_{\perp}$. The directions associated with these modes are plotted in Fig. 2.

In the statistics of the CMB fluctuations, peculiar velocity effects manifest themselves as a set of off-diagonal contributions 


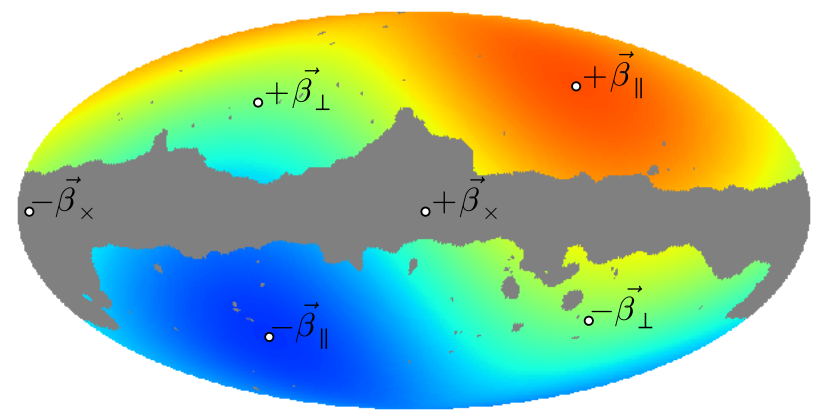

Fig. 2. Specific choice for the decomposition of the dipole vector $\boldsymbol{\beta}$ in Galactic coordinates. The CMB dipole direction $(l, b)=$ $\left(263^{\circ} .99,48^{\circ} .26\right)$ is given as $\beta_{\|}$, while two directions orthogonal to it (and each other) are denoted as $\boldsymbol{\beta}_{\perp}$ and $\boldsymbol{\beta}_{\times}$. The vector $\boldsymbol{\beta}_{\times}$lies within the Galactic plane.

to the $\mathrm{CMB}$ covariance matrix:

$$
\begin{aligned}
\left\langle T_{\ell_{1} m_{2}} T_{\ell_{2} m_{2}}\right\rangle_{\mathrm{CMB}} & =\sum_{L M}(-1)^{M}\left(\begin{array}{ccc}
\ell_{1} & \ell_{2} & L \\
m_{1} & m_{2} & M
\end{array}\right) \\
& \times \sqrt{\frac{\left(2 \ell_{1}+1\right)\left(2 \ell_{2}+1\right)(2 L+1)}{4 \pi}} W_{\ell_{1} \ell_{2} L}^{\beta_{v}} \beta_{L M},
\end{aligned}
$$

where the weight function $W^{\beta_{v}}$ is composed of two parts, related to the aberration and modulation effects, respectively,

$W_{\ell_{1} \ell_{2} L}^{\beta_{v}}=W_{\ell_{1} \ell_{2} L}^{\phi}+b_{v} W_{\ell_{1} \ell_{2} L}^{\tau}$

and the term in large parentheses is a Wigner 3- $j$ symbol. It should be understood that in all of the expressions in this section, we take $L=1$ for our calculations. We have written the expressions in more general form to allow easier connection to more general estimators in the literature. The aberration term, for example, is identical to that found when considering gravitational lensing of the CMB by large-scale structure (Lewis \& Challinor 2006; Planck Collaboration XVII 2014),

$$
\begin{aligned}
W_{\ell_{1} \ell_{2} L}^{\phi}= & -\left(\frac{1+(-1)^{\ell_{1}+\ell_{2}+L}}{2}\right) \sqrt{L(L+1) \ell_{1}\left(\ell_{1}+1\right)} \\
& \times C_{\ell_{1}}^{\mathrm{TT}}\left(\begin{array}{ccc}
\ell_{1} & \ell_{2} & L \\
1 & 0 & -1
\end{array}\right)+\left(\ell_{1} \leftrightarrow \ell_{2}\right),
\end{aligned}
$$

while the modulation term is identical to that produced by an inhomogeneous optical depth to the last scattering surface (Dvorkin \& Smith 2009),

$$
W_{\ell_{1} \ell_{2} L}^{\tau}=\left(\begin{array}{ccc}
\ell_{1} & \ell_{2} & L \\
0 & 0 & 0
\end{array}\right)\left(C_{\ell_{1}}^{\mathrm{TT}}+C_{\ell_{2}}^{\mathrm{TT}}\right) \text {. }
$$

Note that one might, in principle, be concerned about order $\beta^{2}$ corrections to the covariance matrix, particularly at high $\ell$ (see, e.g., Notari \& Quartin 2012). However, these are small, provided that the spectra are relatively smooth. Although the order $\beta\left(\simeq 10^{-3}\right)$ deflections give large changes to the $a_{\ell m}$ s for $\ell>10^{3}$, the changes to the overall covariance are small (Chluba 2011), since the deflection effect is coherent over very large scales.

The basic effect of these boosting-induced correlations is to couple $\ell$ modes with $\ell \pm 1$ modes. They therefore share this property with pure dipolar amplitude modulations studied in the context of primordial statistical anisotropy (see, e.g., Prunet et al. 2005), as well as with dipolar modulations in more general physical parameters (Moss et al. 2011). However, these other cases do not share the frequency dependence of the boosting modulation effect, since they are not accompanied by a temperature dipole.

We measure the peculiar velocity dipole using quadratic estimators, essentially summing over the covariance matrix of the observed $\mathrm{CMB}$ fluctuations, with weights designed to optimally extract $\boldsymbol{\beta}$. A general quadratic estimator $\hat{x}_{L M}$ for $\boldsymbol{\beta}_{L M}$ is given by (Hanson \& Lewis 2009)

$$
\begin{aligned}
\hat{x}_{L M}[\bar{T}]= & \frac{1}{2} N_{L}^{x \beta_{v}} \sum_{\ell_{1}=\ell_{\min }}^{\ell_{\max }} \sum_{\ell_{2}=\ell_{\min }}^{\ell_{\max }} \sum_{m_{1}, m_{2}}(-1)^{M}\left(\begin{array}{ccc}
\ell_{1} & \ell_{2} & L \\
m_{1} & m_{2} & -M
\end{array}\right) W_{\ell_{1} \ell_{2} L}^{x} \\
& \times\left(\bar{T}_{\ell_{1} m_{1}} \bar{T}_{\ell_{2} m_{2}}-\left\langle\bar{T}_{\ell_{1} m_{1}} \bar{T}_{\ell_{2} m_{2}}\right\rangle\right),
\end{aligned}
$$

where $\bar{T}_{\ell m}$ are a set of inverse-variance filtered temperature multipoles, $W_{\ell_{1} \ell_{2} L}^{x}$ is a weight function and $N_{L}^{x \beta_{v}}$ is a normalization. To study the total boosting effect we use Eq. (12) for the weight function, but we will also use weight functions designed to extract specifically the aberration and modulation components of the effect. The ensemble average term \langle\rangle is taken over signal+noise realizations of the $\mathrm{CMB}$ in the absence of velocity effects. It corrects for the statistical anisotropy induced by effects like beam asymmetry, masking, and noise inhomogeneity. We evaluate this term using Monte Carlo simulations of the data, as discussed in Sect. 4.

We use three different quadratic estimators to measure the effects of boosting. The first, $\hat{\beta}$, simply adopts the weight function $W_{\ell_{1} \ell_{2} L}^{\beta_{v}}$, and provides a minimum-variance estimator of the total peculiar velocity effect. The two additional estimators, $\hat{\phi}$ and $\hat{\tau}$, isolate the aberration and modulation aspects of the peculiar velocity effect, respectively. This can be useful, as they are qualitatively quite distinct effects, and suffer from different potential contaminants. The modulation effect, for example, is degenerate with a dipolar pattern of calibration errors on the sky, while the aberration effect is indistinguishable from a dipolar pattern of pointing errors.

There is a subtlety in the construction of these estimators, due to the fact that the covariances, described by $W^{\phi}$ and $W^{\tau}$, are not orthogonal. To truly isolate the aberration and modulation effects, we form orthogonalized weight matrices as

$$
\begin{aligned}
& W_{\ell_{1} \ell_{2} L}^{\hat{\phi}}=W_{\ell_{1} \ell_{2} L}^{\phi}-W_{\ell_{1} \ell_{2} L}^{\tau} \frac{\mathcal{R}_{L}^{\phi \tau}}{\mathcal{R}_{L}^{\tau \tau}} \quad \text { and } \\
& W_{\ell_{1} \ell_{2} L}^{\hat{\tau}}=W_{\ell_{1} \ell_{2} L}^{\tau}-W_{\ell_{1} \ell_{2} L}^{\phi} \frac{\mathcal{R}_{L}^{\tau \phi}}{\mathcal{R}_{L}^{\phi \phi}},
\end{aligned}
$$

where the response function $\mathcal{R}$ is given by

$\mathcal{R}_{L}^{x z}=\frac{1}{(2 L+1)} \sum_{\ell_{1}=\ell_{\min }}^{\ell_{\max }} \sum_{\ell_{2}=\ell_{\min }}^{\ell_{\max }} \frac{1}{2} W_{\ell_{1} \ell_{2} L}^{x} W_{\ell_{1} \ell_{2} L}^{z} F_{\ell_{1}} F_{\ell_{2}}$,

with $x, z=\beta_{v}, \phi, \tau$. The construction of these estimators is analogous to the construction of "bias-hardened" estimators for CMB lensing (Namikawa et al. 2013). The spectra $F_{\ell}$ are diagonal approximations to the inverse variance filter, which takes the sky map $T \rightarrow \bar{T}$. We use the same inverse variance filter as that used for the baseline results in Planck Collaboration XVII (2014), and the approximate filter functions are also specified there. Note that our $\hat{\phi}$ estimator is slightly different from that used in Planck Collaboration XVII (2014), due to the fact that we have orthogonalized it with respect to $\tau$.

The normalization $N_{L}^{x \beta_{v}}$ can be approximated analytically as

$N_{L}^{x \beta_{v}} \simeq\left[\mathcal{R}_{L}^{x \beta_{v}}\right]^{-1}$ 
This approximation does not account for masking. On a masked sky, with this normalization, we expect to find that $\left\langle\hat{x}_{L M}\right\rangle=$ $f_{L M \text {, sky }} \beta_{L M}$, where

$f_{L M, \text { sky }}=\int \mathrm{d} \hat{\boldsymbol{n}} Y_{L M}^{*}(\hat{\boldsymbol{n}}) M(\hat{\boldsymbol{n}}) \boldsymbol{\beta}_{\|} \cdot \hat{\boldsymbol{n}}$.

Here $M(\hat{\boldsymbol{n}})$ is the sky mask used in our analysis. For the fiducial sky mask we use (plotted in Fig. 2, and which leaves approximately $70 \%$ of the sky unmasked), taking the dot product of $f_{1 M \text {, sky }}$ with our three basis vectors we find that $f_{\| \text {, sky }}=0.82$, $f_{\perp \text {, sky }}=0.17$, and $f_{\times \text {, sky }}=-0.04$. The large effective sky fraction for the $\boldsymbol{\beta}_{\|}$direction reflects the fact that the peaks of the expected velocity dipole are untouched by the mask, while the small values of $f_{\text {sky }}$ for the other components reflects that fact that the masking procedure does not leak a large amount of the dipole signal in the $\boldsymbol{\beta}_{\|}$direction into other modes.

\section{Data and simulations}

Given the frequency-dependent nature of the velocity effects we are searching for (at least for the $\tau$ component), we will focus for the most part on estimates of $\beta$ obtained from individual frequency maps, although in Sect. 6 we will also discuss the analysis of component-separated maps obtained from combinations of the entire Planck frequency range. Our analysis procedure is essentially identical to that of Planck Collaboration XVII (2014), and so we only provide a brief review of it here. We use the 143 and $217 \mathrm{GHz}$ Planck maps, which contain the majority of the available CMB signal probed by Planck at the high multipoles required to observe the velocity effects. The $143 \mathrm{GHz}$ map has a noise level that is reasonably well approximated by $45 \mu \mathrm{K}$ arcmin white noise, while the $217 \mathrm{GHz}$ map has approximately twice as much noise power, with a level of $60 \mu \mathrm{K}$ arcmin. The beam at $143 \mathrm{GHz}$ is approximately $7^{\prime}$ FWHM, while the $217 \mathrm{GHz}$ beam is $5^{\prime}$ FWHM. This increased angular resolution, as well as the larger size of the $\tau$-type velocity signal at higher frequency, makes $217 \mathrm{GHz}$ slightly more powerful than $143 \mathrm{GHz}$ for detecting velocity effects (the HFI $100 \mathrm{GHz}$ and LFI $70 \mathrm{GHz}$ channels would offer very little additional constraining power). At these noise levels, for $70 \%$ sky coverage we Fisherforecast a $20 \%$ measurement of the component $\beta_{\|}$at $217 \mathrm{GHz}$ (or, alternatively, a $5 \sigma$ detection) or a $25 \%$ measurement of $\beta_{\|}$at $143 \mathrm{GHz}$, consistent with the estimates of Kosowsky \& Kahniashvili (2011) and Amendola et al. (2011). As we will see, our actual statistical error bars determined from simulations agree well with these expectations.

The Planck maps are generated at HEALPix (Górski et al. $2005)^{3} N_{\text {side }}=2048$. In the process of mapmaking, time-domain observations are binned into pixels. This effectively generates a pointing error, given by the distance between the pixel centre to which each observation is assigned and the true pointing direction at that time. The pixels at $N_{\text {side }}=2048$ have a typical dimension of 1.7. As this is comparable to the size of the aberration effect we are looking for, this is a potential source of concern. However, as discussed in Planck Collaboration XVII (2014), the beam-convolved CMB is sufficiently smooth on these scales that it is well approximated as a gradient across each pixel, and the errors accordingly average down with the distribution of hits in each pixel. For the frequency maps that we use, the rms pixelization error is on the order of 0.1 , and not coherent over the large dipole scales which we are interested in, and so we neglect pixelization effects in our measurement.

3 http://healpix.jpl.nasa.gov
We will use several data combinations to measure $\beta$. The quadratic estimator of Eq. (15) has two input "legs", i.e., the $\ell_{1} m_{1}$ and $\ell_{2} m_{2}$ terms. Starting from the 143 and $217 \mathrm{GHz}$ maps, there are three distinct ways we may source these legs: (1) both legs use either the individually filtered $143 \mathrm{GHz}$ or $217 \mathrm{GHz}$ maps, which we refer to as $143 \times 143$ and $217 \times 217$, respectively; (2) we can use $143 \mathrm{GHz}$ for one leg, and $217 \mathrm{GHz}$ for the other, referred to as $143 \times 217$; and (3) we can combine both 143 and $217 \mathrm{GHz}$ data in our inverse-variance filtering procedure into a single minimum-variance map, which is then fed into both legs of the quadratic estimator. We refer to this final combination schematically as " $143+217$ ". Combinations (2) and (3) mix 143 and $217 \mathrm{GHz}$ data. When constructing the weight function of Eq. (12) for these combinations we use an effective $b_{v}=2.5$. Note that this effective $b_{v}$ is only used to determine the weight function of the quadratic estimator; errors in the approximation will make our estimator suboptimal, but will not bias our results. To construct the $\bar{T}$, which are the inputs for these quadratic estimators, we use the filtering described in Appendix A of Planck Collaboration XVII (2014), which optimally accounts for the Galactic and point source masking (although not for the inhomogeneity of the instrumental noise level). This filter inversevariance weights the CMB fluctuations, and also projects out the $857 \mathrm{GHz}$ Planck map as a dust template.

To characterize our estimator and to compute the mean-field term of Eq. (15), we use a large set of Monte Carlo simulations. These are generated following the same procedure as those described in Planck Collaboration XVII (2014); they incorporate the asymmetry of the instrumental beam, gravitational lensing effects, and realistic noise realizations from the FFP6 simulation set described in Planck Collaboration I (2014) and Planck Collaboration (2013). There is one missing aspect of these simulations which we discuss briefly here: due to an oversight in their preparation, the gravitational lensing component of our simulations only included lensing power for lensing modes on scales $L \geq 2$, which leads to a slight underestimation of our simulationbased error bars for the $\phi$ component of the velocity estimator. The lensing dipole power in the fiducial $\Lambda \mathrm{CDM}$ model is $C_{1}^{\phi \phi} \simeq 6 \times 10^{-8}$, which represents an additional source of noise for each mode of $\beta$, given by $\sigma_{\phi \text {, lens }}=1.2 \times 10^{-4}$, or about one tenth the size of the expected signal. The $\phi$ part of the estimator contributes approximately $46 \%$ of the total $\beta$ estimator weight at $143 \mathrm{GHz}$, and $35 \%$ at $217 \mathrm{GHz}$. Our measurement errors without this lensing noise on an individual mode of $\beta$ are $\sigma_{\beta} \simeq 2.5 \times 10^{-4}$, while with lensing noise included we would expect this to increase to $\sqrt{\sigma_{\beta}^{2}+(4 / 10)^{2} \sigma_{\phi, \text { lens }}^{2}}=2.54 \times 10^{-4}$. This is small enough that we have neglected it for these results (rather than include it by hand).

We generate simulations both with and without peculiar velocity effects, to determine the normalization of our estimator, which, as we will see, is reasonably consistent with the analytical expectation discussed around Eq. (20). All of our main results with frequency maps use 1000 simulations to determine the estimator mean field and variance, while the component separation tests in Sect. 6 use 300 simulations.

\section{Results}

We present our results visually in Fig. 3, where we plot the total measured dipole direction $\hat{\boldsymbol{\beta}}$ as a function of the maximum temperature multipole $\ell_{\max }$ used as input to our quadratic estimators. We can see that all four of our $143 / 217 \mathrm{GHz}$ based estimators converge towards the expected dipole direction at high $\ell_{\max }$. 

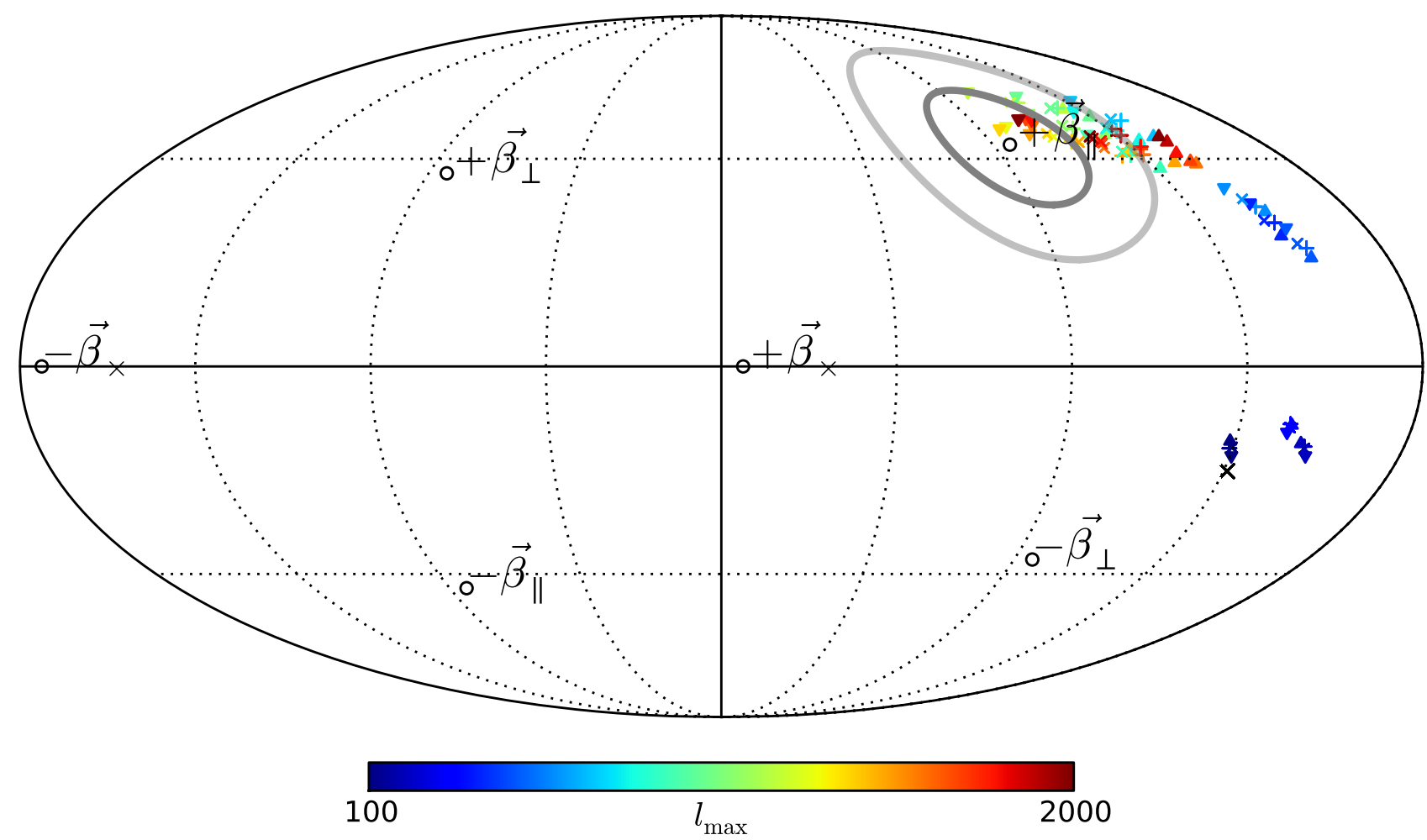

Fig. 3. Measured dipole direction $\hat{\boldsymbol{\beta}}$ in Galactic coordinates as a function of the maximum temperature multipole used in the analysis, $\ell_{\max }$. We plot the results for the four data combinations discussed in Sect. 4: $143 \times 143(\boldsymbol{\nabla}$ symbol); $217 \times 217(\boldsymbol{\Lambda}$ symbol); $143 \times 217(\times$ symbol); and $143+217$ (+ symbol). The CMB dipole direction $\boldsymbol{\beta}_{\|}$has been highlighted with $14^{\circ}$ and $26^{\circ}$ radius circles, which correspond roughly to our expected uncertainty on the dipole direction. The black cross in the lower hemisphere is the modulation dipole anomaly direction found for $W M A P$ at $\ell_{\max }=64$ in Hoftuft et al. (2009), and which is discussed further in Planck Collaboration XXIII (2014). Note that all four estimators are significantly correlated with one another, even the $143 \times 143$ and $217 \times 217$ results, which are based on maps with independent noise realizations. This is because a significant portion of the dipole measurement uncertainty is from sample variance of the CMB fluctuations, which is common between channels.

At $\ell_{\max }<100$, we recover the significantly preferred direction of Hoftuft et al. (2009), which is identified when searching for a dipolar modulation of the CMB fluctuations (see Planck Collaboration XXIII 2014). The $\tau$ component of the velocity effect is degenerate with such a modulation (at least at fixed frequency), and $\phi$ gets little weight from $\ell<100$, so this is an expected result. The significance of this preferred direction varies as a function of smoothing scale (Hanson \& Lewis 2009; Bennett et al. 2011; Planck Collaboration XXIII 2014). To minimize possible contamination of our results by this potential anomaly, from here onward we restrict the temperature multipoles used in our $\beta$ estimation to $\ell_{\min }=500$. This cut removes only about $10 \%$ of the total number of modes measured by Planck, and so does not significantly increase our error bars. Note also that we have verified that our error bars do not shrink significantly for $\ell_{\max }>2000$, since almost all of the modes measured by Planck are at $\ell<2000$.

There is a clear tendency in Fig. 3 for the measured velocity to point towards the expected direction $\boldsymbol{\beta}_{\|}$. At Planck noise levels, we expect a $1 \sigma$ uncertainty on each component of $\boldsymbol{\beta}$ of better than $25 \%$. A $25 \%$ uncertainty corresponds to an $\arctan (1 / 4)=$ $14^{\circ}$ constraint on the direction of $\beta$. We plot this contour, as well as the corresponding $2 \sigma$ contour $\arctan (2 / 4)=26^{\circ}$. It is apparent that the measured velocity directions are in reasonable agreement with the $\mathrm{CMB}$ dipole.

We now proceed to break the measurement of Fig. 3 into its constituent parts for $\ell_{\max }=2000$ (and truncating now at $\left.\ell_{\min }=500\right)$. In Fig. 4 we plot our quadratic estimates of the three components of $\boldsymbol{\beta}$, as well as the decomposition into aberration and modulation components, for each of our four frequency combinations. The vertical lines in Fig. 4 give the amplitude estimates for each component measured from the data, while the coloured and grey histograms give the distribution of these quantities for the $143 \times 217$ estimator, for simulations with and without velocity effects, respectively (the other estimators are similar). As expected, the velocity effects show up primarily in $\beta_{\|}$; there is little leakage into other components with our sky mask. For all four estimators, we see that the presence of velocity along $\boldsymbol{\beta}_{\|}$is strongly preferred over the null hypothesis. At $143 \mathrm{GHz}$ this signal comes from both $\hat{\phi}_{\|}$and $\hat{\tau}_{\|}$. At $217 \mathrm{GHz}$ it comes primarily from $\hat{\tau}_{\|}$. Additionally, there is a somewhat unexpected signal at $217 \mathrm{GHz}$ in the $\boldsymbol{\beta}_{\times}$direction, again driven by the $\tau$ component. Given the apparent frequency dependence, foreground contamination seems a possible candidate for this anomalous signal. We will discuss this possibility further in the next section.

In Table 1 we present $\chi^{2}$ values for the $\beta$ measurements of Fig. 4 under both the null hypothesis of no velocity effects, and assuming the expected velocity direction and amplitude. We can see that all of our measurements are in significant disagreement with the "no velocity" hypothesis. The probability-to-exceed (PTE) values for the "with velocity" case are much more reasonable. Under the velocity hypothesis, $217 \times 217$ has the lowest PTE, of $11 \%$, driven by the large $\hat{\beta}_{\times}$.

In Table 2 we focus on our measurements of the velocity amplitude along the expected direction $\boldsymbol{\beta}_{\|}$, as well as performing null tests among our collection of estimates. For this table, we have normalized the estimators, such that the average of $\hat{\beta}_{\|}$on boosted simulations is equal to the input value 

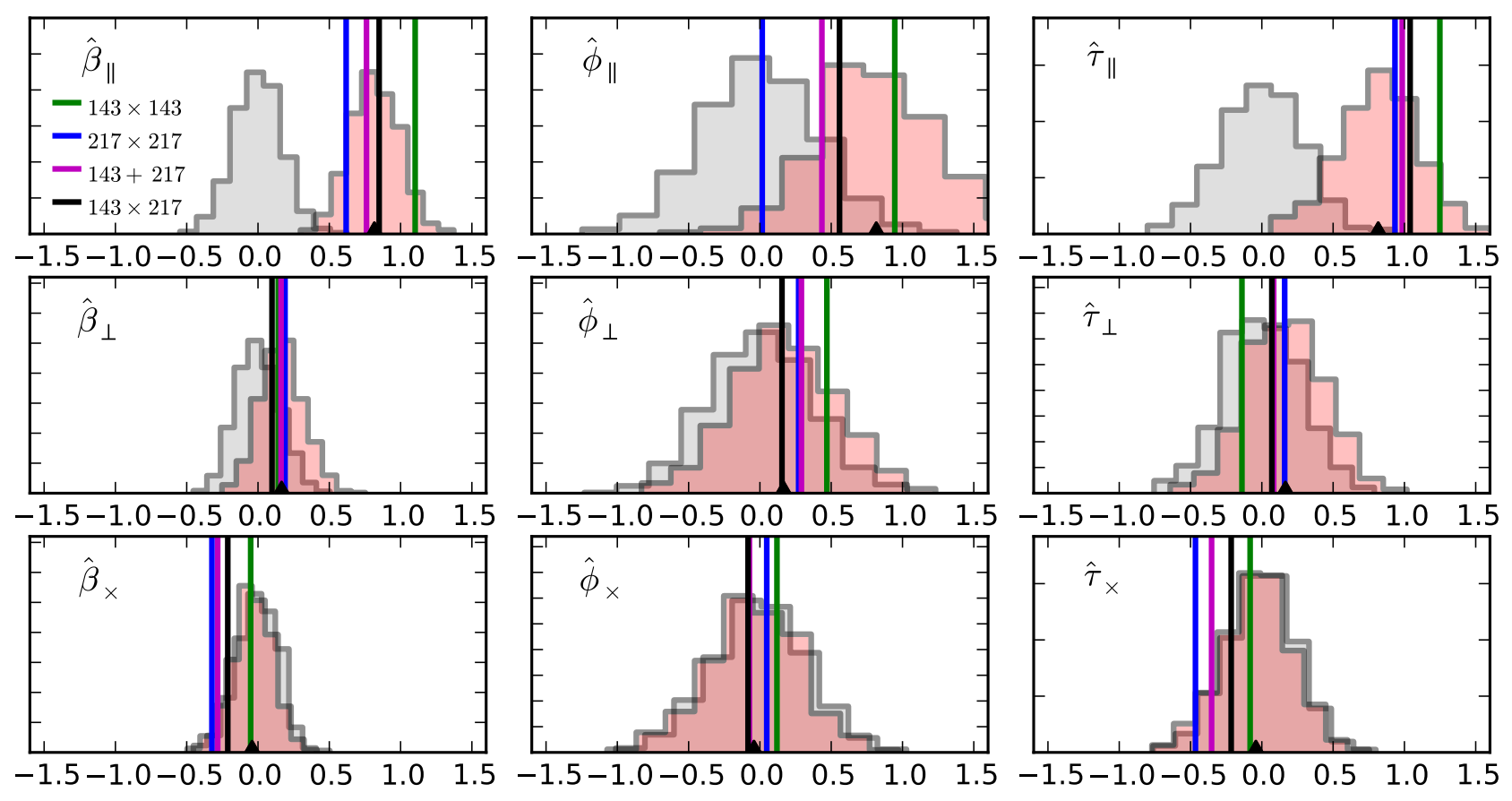

Fig. 4. Measurements of $\beta$ using combinations of the 143 and $217 \mathrm{GHz}$ Planck maps, normalized using Eq. (19) and then divided by the fiducial amplitude of $\beta=1.23 \times 10^{-3}$. These estimates use $\ell_{\min }=500$ and $\ell_{\max }=2000$. In addition to the total minimum variance estimate $\hat{\beta}$, the measurement is also broken down into its aberration-type part, $\hat{\phi}$, and modulation-type part, $\hat{\tau}$. Vertical lines give the Planck measurement for the four estimates described in the text. Grey histograms give the distribution of estimates for simulations of the $143 \times 217$ estimator, which do not contain peculiar velocity effects (the other estimators are very similar). The red histograms give the distribution for simulations which do contain peculiar velocity effects, simulated with the fiducial direction (along $\boldsymbol{\beta}_{\|}$) and amplitude. Black triangles on the $x$-axis indicate the relevant component of $f_{\text {sky }}$ given by Eq. (20), which agrees well with the peak of the velocity simulations.

Table 1. Significance measures for the $\boldsymbol{\beta}$ estimates of Fig. 4.

\begin{tabular}{ccccc}
\hline \hline & \multicolumn{3}{c}{$\hat{\beta}$ Significance } \\
\cline { 2 - 5 } & \multicolumn{2}{c}{ No velocity } & \multicolumn{2}{c}{ With velocity } \\
\hline & $\chi^{2}$ & PTE [\%] & $\chi^{2}$ & PTE [\%] \\
\hline $143 \times 143 \ldots$ & 27.1 & 0.0005 & 1.9 & 58.87 \\
$217 \times 217 \ldots$ & 20.7 & 0.0123 & 6.0 & 11.18 \\
$143+217 \ldots$ & 25.1 & 0.0015 & 3.3 & 35.44 \\
$143 \times 217 \ldots$ & 27.6 & 0.0005 & 1.8 & 62.29 \\
\hline
\end{tabular}

Notes. We form a $\chi^{2}$ for the three measured modes of $\beta$, using the mean and covariance matrix measured for simulations either with or without velocity effects. The covariance matrices are very similar in both cases, and so the difference between these two cases is only the mean-field subtraction. The "PTE" columns give the corresponding probabilityto-exceed values for a $\chi^{2}$ distribution with three degrees of freedom. The measured $\chi^{2}$ values are extremely unlikely under the "no velocity" hypothesis, but very compatible for the "with velocity" case.

of $369 \mathrm{~km} \mathrm{~s}^{-1}$. For all four of our estimators, we find that this normalization factor is within $0.5 \%$ of that given by $N^{x \beta_{v}} f_{\|, \mathrm{sky}}$, as is already apparent from the triangles along the horizontal axis of Fig. 4. We can see here, as expected, that our estimators have a statistical uncertainty on $\beta_{\|}$of between $20 \%$ and $25 \%$. However, several of our null tests, obtained by taking the differences of pairs of $\beta_{\|}$estimates, fail at the level of $2-3 \sigma$. We take the $143 \times 217 \mathrm{GHz}$ estimator as our fiducial measurement; because it involves the cross-correlation of two maps with independent noise realizations it should be robust
Table 2. Measured velocity amplitudes along the $\boldsymbol{\beta}_{\|}$direction, in units of $369 \mathrm{~km} \mathrm{~s}^{-1}$, using combinations of the 143 and $217 \mathrm{GHz}$ data, as discussed in Sect. 4.

\begin{tabular}{|c|c|c|c|c|}
\hline & \multicolumn{4}{|c|}{$\hat{\beta}_{\|}$Amplitude } \\
\hline & $143 \times 143$ & $217 \times 217$ & $143+217$ & $143 \times 217$ \\
\hline $143 \times 143$ & $1.35 \pm 0.26$ & & & \\
\hline $217 \times 217$ & $0.60 \pm 0.21$ & $0.75 \pm 0.19$ & & \\
\hline $143+217$ & $0.43 \pm 0.15$ & $-0.17 \pm 0.09$ & $0.92 \pm 0.20$ & \\
\hline $143 \times 217$ & $0.31 \pm 0.13$ & $-0.29 \pm 0.12$ & $-0.12 \pm 0.07$ & $1.04 \pm 0.21$ \\
\hline
\end{tabular}

Notes. The diagonal shows the results for the indicated reconstruction. Below the diagonal, the numbers given are for the difference of the two results, and the uncertainty accounts for the correlation between each pair of measurements. This lower triangle is a null test. Several of these null tests fail between 143 and $217 \mathrm{GHz}$, although both channels provide evidence for $\beta_{\|}$, which is consistent with the expected amplitude and discrepant with zero at $4-5 \sigma$.

to noise modelling. Null tests against the individual 143 and $217 \mathrm{GHz}$ estimates are in tension at a level of $2 \sigma$ for this estimator. We take this tension as a measure of the systematic differences between these two channels, and conservatively choose the largest discrepancy with the $143 \times 217 \mathrm{GHz}$ estimate, namely 0.31 , as our systematic error. We therefore report a measurement of $\hat{v}_{\|}=384 \mathrm{~km} \mathrm{~s}^{-1} \pm 78 \mathrm{~km} \mathrm{~s}^{-1}$ (stat.) $\pm 115 \mathrm{~km} \mathrm{~s}^{-1}$ (syst.), a significant confirmation of the expected velocity amplitude. 

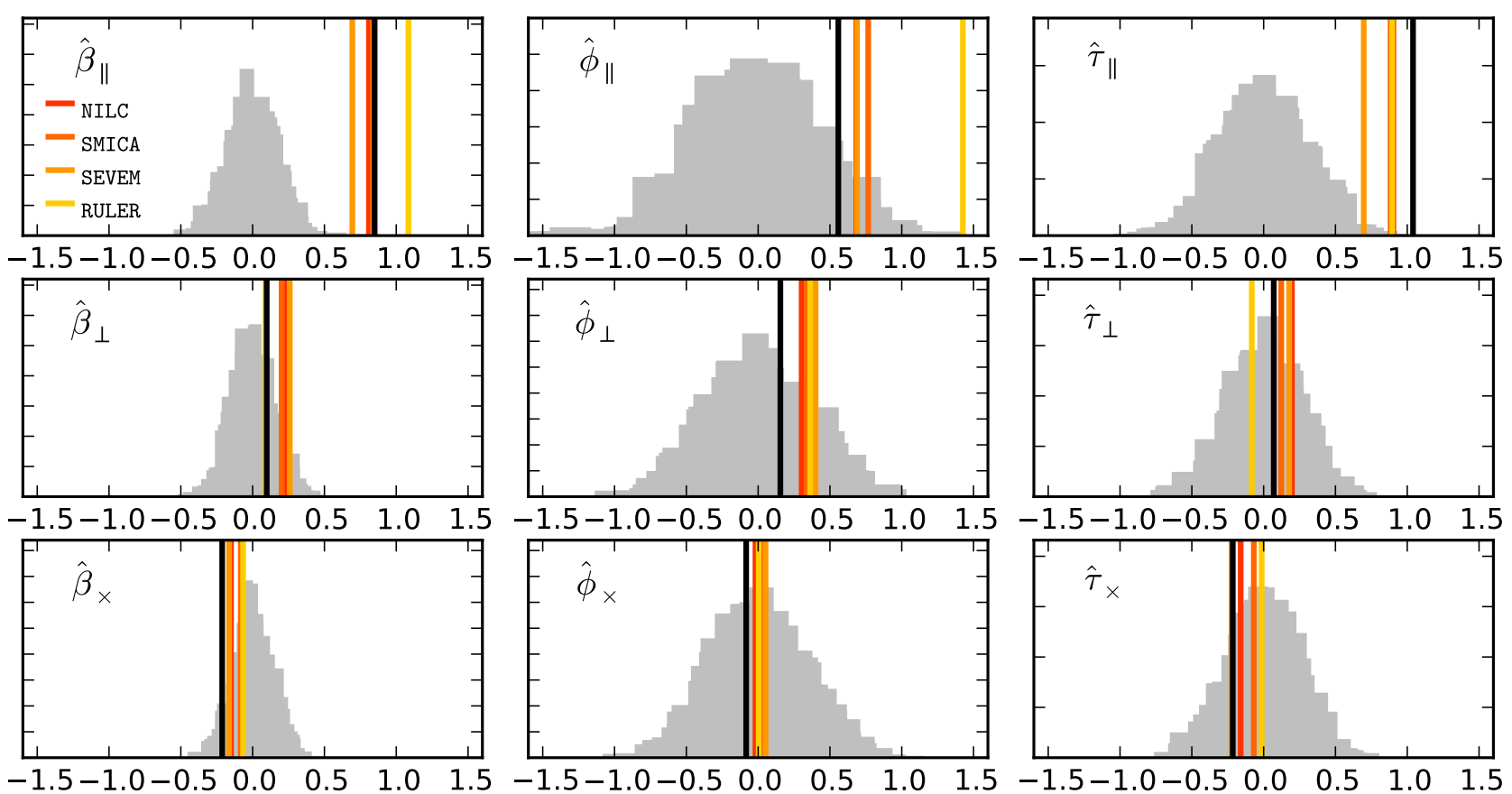

Fig. 5. Plot of velocity amplitude estimates, similar to Fig. 4, but using an array of component-separated maps, rather than specific combinations of frequency maps. The production and characterization of these component-separated maps is presented in Planck Collaboration XII (2014). Histograms of simulation results without velocity effects are overplotted in grey for each method; they are all very similar. Vertical coloured bars correspond to the maps indicated in the legend, using the combination of our fiducial galaxy mask (which removes approximately $30 \%$ of the sky), as well as the specific mask produced for each component separation method. We see significant departures from the null-hypothesis simulations only in the $\boldsymbol{\beta}_{\|}$direction, as expected. Vertical black lines show the $143 \times 217$ measurement of Fig. 4 . Note the discussion about the subtleties in the normalization of these estimates in Sect. 6.

\section{Potential contaminants}

There are several potential sources of contamination for our estimates above which we discuss briefly here, although we have not attempted an exhaustive study of potential contaminants for our estimator.

Galactic Foregrounds: given the simplicity of the foreground correction we have used (consisting only of masking the sky and projecting out the Planck $857 \mathrm{GHz}$ map as a crude dust template), foreground contamination is a clear source of concern. The frequency dependence of the large $\beta_{\times}$signal seen at $217 \mathrm{GHz}$, but not at $143 \mathrm{GHz}$, seems potentially indicative of foreground contamination, as the Galactic dust power is approximately 10 times larger at 217 than at $143 \mathrm{GHz}$. To test the possible magnitude of residual foregrounds, we apply our velocity estimators to the four component-separated CMB maps of Planck Collaboration XII (2014), i.e., NILC, SMICA, SEVEM, and COMMANDER-RULER. Each of these methods combines the full set of nine Planck frequency maps from 30 to $857 \mathrm{GHz}$ to obtain a best-estimate CMB map. To characterize the scatter and mean field of each method's map we use the set of common simulations which each method has been applied to. These simulations include the effect of the aberration part of the velocity dipole, although not the frequency-dependent modulation part. For this reason, it is difficult to accurately assess the normalization of our estimators when applied to these maps, particularly as they can mix 143 and $217 \mathrm{GHz}$ as a function of scale, and the modulation part is frequency dependent. We can, however, study them at a qualitative level. The results of this analysis are shown in Fig. 5. To construct our $\boldsymbol{\beta}$ estimator for the component-separated maps we have used $b_{v}=2.5$, assuming that they contain roughly equal contributions from 143 and $217 \mathrm{GHz}$. Note that, because the simulations used to determine the mean fields of the componentseparated map included the aberration part of the velocity effect, it will be absorbed into the mean field if uncorrected. Because the aberration contribution is frequency independent (so there are no issues with how the different CMB channels are mixed), and given the good agreement between our analytical normalization and that measured using simulations for the frequency maps, when generating Fig. 5 we have subtracted the expected velocity contribution from the mean field analytically. We see generally good agreement with the $143 \times 217$ estimate on which we have based our measurement of the previous section; there are no obvious discrepancies with our measurements in the $\boldsymbol{\beta}_{\|}$direction, although there is a somewhat large scatter between methods for $\hat{\phi}_{\|}$. In the $\boldsymbol{\beta}_{\times}$direction the component-separated map estimates agree well with the $143 \times 217$ estimator, and do not show the significant power seen for $217 \times 217$, suggesting that the large power that we see there may indeed be foreground in origin.

Calibration errors: position-dependent calibration errors in our sky maps are completely degenerate with modulation-type effects (at fixed frequency), and so are very worrisome as a potential systematic effect. We note that the Planck scan strategy strongly suppresses map calibration errors with large-scale structure (such as a dipole, see Planck Collaboration VIII 2014). As the satellite spins, the detectors mounted in the focal plane inscribe circles on the sky, with opening angles of between $83^{\circ}$ and $85^{\circ}$ (for the 143 and $217 \mathrm{GHz}$ detectors we use). For a time-dependent calibration error to project cleanly into a dipolar structure on the sky, it would need to have a periodicity comparable to the spin frequency of the satellite $\left(1 \mathrm{~min}^{-1}\right)$. Slower fluctuations in the calibration should be strongly suppressed in the maps. There are calibration errors which are not suppressed 
by the scan strategy, however. For example, a nonlinear detector response could couple directly to the large CMB dipole temperature. Ultimately, because position-dependent calibration errors are completely degenerate with the $\tau$ component of the velocity effect, the only handle which we have on them for this study is the consistency between $\hat{\phi}$ and $\hat{\tau}$. From another viewpoint, the consistency of our measurement with the expected velocity modulation provides an upper bound on dipolar calibration errors.

Pointing errors: in principle, errors in the pointing are perfectly degenerate with the aberration-type velocity effect in the observed CMB. However the Planck pointing solution has an uncertainty of a few arcseconds rms in both the co- and cross-scan directions (Planck Collaboration VI 2014). The 3' rms aberrations induced by velocity effects are simply too large to be contaminated by any reasonable pointing errors.

\section{Conclusions}

From Fig. 3 it is clear that small-scale CMB fluctuations observed in the Planck data provide evidence for velocity effects in the expected direction. This is put on more quantitative footing in Fig. 4 and Table 2, where we see that all four of the 143 and $217 \mathrm{GHz}$ velocity estimators which we have considered show evidence for velocity effects along $\boldsymbol{\beta}_{\|}$at above the $4 \sigma$ level. Detailed comparison of 143 and $217 \mathrm{GHz}$ data shows some discrepancies, which we have taken as part of a systematic error budget; however, tests with component-separated maps shown in Fig. 5 provide a strong indication that our $217 \mathrm{GHz}$ map has slight residual foreground contamination. The componentseparated results are completely consistent with the $143 \times 217$ estimator which we quote for our fiducial result.

Beyond our peculiar velocity's impact on the CMB, there have been many studies of related effects at other wavelengths (e.g., Blake \& Wall 2002; Titov et al. 2011; Gibelyou \& Huterer 2012). Closely connected are observational studies which examine the convergence of the clustering dipole (e.g., Itoh et al. 2010; Bilicki et al. 2011). Indications of non-convergence might be evidence for a super-Hubble isocurvature mode, which can generate a "tilt" between matter and radiation (Turner 1991), leading to an extremely large-scale bulk flow. Such a longwavelength isocurvature mode could also contribute a significant "intrinsic" component to our observed temperature dipole (Langlois \& Piran 1996). However, a peculiar velocity dipole is expected at the level of $\sqrt{C_{1}} \sim 10^{-3}$ due to structure in standard $\Lambda$ CDM (see, e.g., Zibin \& Scott 2008), which suggests that an intrinsic component, if it exists at all, is subdominant. In addition, such a bulk flow has been significantly constrained by Planck studies of the kinetic Sunyaev-Zeldovich effect (Planck Collaboration Int. XIII 2014). In this light, the observation of aberration at the expected level reported in this paper is fully consistent with the standard, adiabatic picture of the Universe.

The Copernican revolution taught us to see the Earth as orbiting a stationary Sun. That picture was eventually refined to include Galactic and cosmological motions of the Solar System. Because of the technical challenges, one may have thought it very unlikely to be able to measure (or perhaps even to define) the cosmological motion of the Solar System ... and yet it moves.

Acknowledgements. The development of Planck has been supported by: ESA; CNES and CNRS/INSU-IN2P3-INP (France); ASI, CNR, and INAF (Italy); NASA and DoE (USA); STFC and UKSA (UK); CSIC, MICINN, JA, and RES (Spain); Tekes, AoF, and CSC (Finland); DLR and MPG (Germany); CSA (Canada); DTU Space (Denmark); SER/SSO (Switzerland); RCN (Norway); SFI (Ireland); FCT/MCTES (Portugal); and PRACE (EU).
A description of the Planck Collaboration and a list of its members, including the technical or scientific activities in which they have been involved, can be found at http://www.sciops.esa.int/index.php? project=planck\&page $=\mathrm{Pl}$ lanck_Collaboration. Some of the results in this paper have been derived using the HEALPix package. This research used resources of the National Energy Research Scientific Computing Center, which is supported by the Office of Science of the US Department of Energy under Contract No. DE-AC02-05CH11231. We acknowledge support from the Science and Technology Facilities Council [grant number ST/I000976/1].

\section{References}

Amendola, L., Catena, R., Masina, I., et al. 2011, JCAP, 1107, 027 Bennett, C., Hill, R., Hinshaw, G., et al. 2011, ApJS, 192, 17 Bilicki, M., Chodorowski, M., Jarrett, T., \& Mamon, G. A. 2011, ApJ, 741, 31 Blake, C., \& Wall, J. 2002, Nature, 416, 150

Burles, S., \& Rappaport, S. 2006, ApJ, 641, L1

Catena, R., Liguori, M., Notari, A., \& Renzi, A. 2013, J. Cosmol. Astropart., 9, 36

Catena, R., \& Notari, A. 2013, J. Cosmol. Astropart., 4, 28

Challinor, A., \& van Leeuwen, F. 2002, Phys. Rev., D65, 103001

Chluba, J. 2011, MNRAS, 415, 3227

Dvorkin, C., \& Smith, K. M. 2009, Phys. Rev., D79, 043003

Eisner, E. 1967, Am. J. Phys., 35, 817

Fixsen, D. J. 2009, ApJ, 707, 916

Fixsen, D., Cheng, E., Gales, J., et al. 1996, ApJ, 473, 576

Gibelyou, C., \& Huterer, D. 2012, MNRAS, 427, 1994

Górski, K. M., Hivon, E., Banday, A. J., et al. 2005, ApJ, 622, 759

Hanson, D., \& Lewis, A. 2009, Phys. Rev., D80, 3004

Hinshaw, G., Weiland, J. L., Hill, R. S., et al. 2009, ApJS, 180, 225

Hoftuft, J., Eriksen, H., Banday, A., et al. 2009, ApJ, 699, 985

Itoh, Y., Yahata, K., \& Takada, M. 2010, Phys. Rev. D, 82, 043530

Kamionkowski, M., \& Knox, L. 2003, Phys. Rev. D, 67, 3001

Kogut, A., Lineweaver, C., Smoot, G. F., et al. 1993, ApJ, 419, 1

Kosowsky, A., \& Kahniashvili, T. 2011, Phys. Rev. Lett., 106, 1301

Langlois, D., \& Piran, T. 1996, Phys. Rev. D, 53, 2908

Lewis, A., \& Challinor, A. 2006, Phys. Rept., 429, 1

Moss, A., Scott, D., Zibin, J. P., \& Battye, R. 2011, Phys. Rev. D, 84, 3014

Namikawa, T., Hanson, D., \& Takahashi, R. 2013, MNRAS, 431, 609

Notari, A., \& Quartin, M. 2012, JCAP, 1202, 026

Pereira, T. S., Yoho, A., Stuke, M., \& Starkman, G. D. 2010 [arXiv: 1009.4937]

Phipps, T. E. 1989, Am. J. Phys., 57, 549

Planck Collaboration 2013, The Explanatory Supplement to the Planck 2013 results, http://www.sciops.esa.int/wikiSI/planckpla/index.php? title=Main_Page (ESA)

Planck Collaboration I. 2014, A\&A, 571, A1

Planck Collaboration II. 2014, A\&A, 571, A2

Planck Collaboration III. 2014, A\&A, 571, A3

Planck Collaboration IV. 2014, A\&A, 571, A4

Planck Collaboration V. 2014, A\&A, 571, A5

Planck Collaboration VI. 2014, A\&A, 571, A6

Planck Collaboration VII. 2014, A\&A, 571, A7

Planck Collaboration VIII. 2014, A\&A, 571, A8

Planck Collaboration IX. 2014, A\&A, 571, A9

Planck Collaboration X. 2014, A\&A, 571, A10

Planck Collaboration XI. 2014, A\&A, 571, A11

Planck Collaboration XII. 2014, A\&A, 571, A12

Planck Collaboration XIII. 2014, A\&A, 571, A13

Planck Collaboration XIV. 2014, A\&A, 571, A14

Planck Collaboration XV. 2014, A\&A, 571, A15

Planck Collaboration XVI. 2014, A\&A, 571, A16

Planck Collaboration XVII. 2014, A\&A, 571, A17

Planck Collaboration XVIII. 2014, A\&A, 571, A18

Planck Collaboration XIX. 2014, A\&A, 571, A19

Planck Collaboration XX. 2014, A\&A, 571, A20

Planck Collaboration XXI. 2014, A\&A, 571, A21

Planck Collaboration XXII. 2014, A\&A, 571, A22

Planck Collaboration XXIII. 2014, A\&A, 571, A23

Planck Collaboration XXIV. 2014, A\&A, 571, A24

Planck Collaboration XXV. 2014, A\&A, 571, A25

Planck Collaboration XXVI. 2014, A\&A, 571, A26

Planck Collaboration XXVII. 2014, A\&A, 571, A27

Planck Collaboration XXVIII. 2014, A\&A, 571, A28

Planck Collaboration XXIX. 2014, A\&A, 571, A29

Planck Collaboration XXX. 2014, A\&A, 571, A30

Planck Collaboration XXXI. 2014, A\&A, 571, A31 
Planck Collaboration Int. XIII. 2014, A\&A, 561, A97

Prunet, S., Uzan, J.-P., Bernardeau, F., \& Brunier, T. 2005, Phys. Rev. D, 71, 3508

Sollom, I. 2010, Ph.D. thesis, University of Cambridge, UK

Titov, O., Lambert, S. B., \& Gontier, A.-M. 2011, A\&A, 529, A91

Turner, M. S. 1991, Phys. Rev. D, 44, 3737

Yoho, A., Copi, C. J., Starkman, G. D., \& Pereira, T. S. 2012 [arXiv: 1211.6756]

Zibin, J. P., \& Scott, D. 2008, Phys. Rev. D, 78, 3529

1 APC, AstroParticule et Cosmologie, Université Paris Diderot, CNRS/IN2P3, CEA/lrfu, Observatoire de Paris, Sorbonne Paris Cité, 10 rue Alice Domon et Léonie Duquet, 75205 Paris Cedex 13, France

2 Aalto University Metsähovi Radio Observatory and Dept of Radio Science and Engineering, PO Box 13000, 00076 Aalto, Finland

3 African Institute for Mathematical Sciences, 6-8 Melrose Road, 7945 Muizenberg, Cape Town, South Africa

4 Agenzia Spaziale Italiana Science Data Center, via del Politecnico snc, 00133 Roma, Italy

5 Agenzia Spaziale Italiana, Viale Liegi 26, 00198 Roma, Italy

6 Astrophysics Group, Cavendish Laboratory, University of Cambridge, J J Thomson Avenue, Cambridge CB3 0HE, UK

7 Astrophysics \& Cosmology Research Unit, School of Mathematics, Statistics \& Computer Science, University of KwaZulu-Natal, Westville Campus, Private Bag X54001, 4000 Durban, South Africa

8 CITA, University of Toronto, 60 St. George St., Toronto, ON M5S 3H8, Canada

9 CNRS, IRAP, 9 Av. colonel Roche, BP 44346, 31028 Toulouse Cedex 4, France

10 California Institute of Technology, Pasadena, California, USA

11 Centre for Theoretical Cosmology, DAMTP, University of Cambridge, Wilberforce Road, Cambridge CB3 0WA, UK

12 Computational Cosmology Center, Lawrence Berkeley National Laboratory, Berkeley, California, USA

13 DSM/Irfu/SPP, CEA-Saclay, 91191 Gif-sur-Yvette Cedex, France

14 DTU Space, National Space Institute, Technical University of Denmark, Elektrovej 327, 2800 Kgs. Lyngby, Denmark

15 Département de Physique Théorique, Université de Genève, 24 quai E. Ansermet, 1211 Genève 4, Switzerland

16 Departamento de Física Fundamental, Facultad de Ciencias, Universidad de Salamanca, 37008 Salamanca, Spain

17 Departamento de Física, Universidad de Oviedo, Avda. Calvo Sotelo s/n, 33007 Oviedo, Spain

18 Department of Astrophysics/IMAPP, Radboud University Nijmegen, PO Box 9010, 6500 GL Nijmegen, The Netherlands

19 Department of Electrical Engineering and Computer Sciences, University of California, Berkeley, California, USA

20 Department of Physics \& Astronomy, University of British Columbia, 6224 Agricultural Road, Vancouver, British Columbia, Canada

21 Department of Physics and Astronomy, Dana and David Dornsife College of Letter, Arts and Sciences, University of Southern California, Los Angeles, CA 90089, USA

22 Department of Physics and Astronomy, University College London, London WC1E 6BT, UK

23 Department of Physics and Astronomy, University of Sussex, Brighton BN1 9QH, UK

24 Department of Physics, Florida State University, Keen Physics Building, 77 Chieftan Way, Tallahassee, Florida, USA

25 Department of Physics, Gustaf Hällströmin katu 2a, University of Helsinki, 00014 Helsinki, Finland

26 Department of Physics, Princeton University, Princeton, New Jersey, USA

27 Department of Physics, University of California, Berkeley, California, USA

28 Department of Physics, University of California, One Shields Avenue, Davis, California, USA

29 Department of Physics, University of California, Santa Barbara, California, USA
30 Department of Physics, University of Illinois at Urbana-Champaign, 1110 West Green Street, Urbana, Illinois, USA

31 Dipartimento di Fisica e Astronomia G. Galilei, Università degli Studi di Padova, via Marzolo 8, 35131 Padova, Italy

32 Dipartimento di Fisica e Scienze della Terra, Università di Ferrara, via Saragat 1, 44122 Ferrara, Italy

33 Dipartimento di Fisica, Università La Sapienza, P. le A. Moro 2, 00185 Roma, Italy

34 Dipartimento di Fisica, Università degli Studi di Milano, via Celoria, 16, 20133 Milano, Italy

35 Dipartimento di Fisica, Università degli Studi di Trieste, via A. Valerio 2, 34127 Trieste, Italy

36 Dipartimento di Fisica, Università di Roma Tor Vergata, via della Ricerca Scientifica, 1, 00133 Roma, Italy

37 Discovery Center, Niels Bohr Institute, Blegdamsvej 17, 2100 Copenhagen, Denmark

38 Dpto. Astrofísica, Universidad de La Laguna (ULL), 38206 La Laguna, Tenerife, Spain

39 European Space Agency, ESAC, Planck Science Office, Camino bajo del Castillo, s/n, Urbanización Villafranca del Castillo, Villanueva de la Cañada, 28692 Madrid, Spain

40 European Space Agency, ESTEC, Keplerlaan 1, 2201 AZ Noordwijk, The Netherlands

41 Helsinki Institute of Physics, Gustaf Hällströmin katu 2, University of Helsinki, 00014 Helsinki, Finland

42 INAF - Osservatorio Astronomico di Padova, Vicolo dell'Osservatorio 5, 35122 Padova, Italy

43 INAF - Osservatorio Astronomico di Roma, via di Frascati 33, 00040 Monte Porzio Catone, Italy

44 INAF - Osservatorio Astronomico di Trieste, via G.B. Tiepolo 11, 34143 Trieste, Italy

45 INAF Istituto di Radioastronomia, via P. Gobetti 101, 40129 Bologna, Italy

46 INAF/IASF Bologna, via Gobetti 101, 20133 Bologna, Italy

47 INAF/IASF Milano, via E. Bassini 15, Milano, Italy

48 INFN, Sezione di Bologna, via Irnerio 46, 40126 Bologna, Italy

49 INFN, Sezione di Roma 1, Università di Roma Sapienza, Piazzale Aldo Moro 2, 00185 Roma, Italy

50 INFN/National Institute for Nuclear Physics, via Valerio 2, 34127 Trieste, Italy

51 IPAG: Institut de Planétologie et d'Astrophysique de Grenoble, Université Joseph Fourier, Grenoble 1/CNRS-INSU, UMR 5274, 38041 Grenoble, France

52 ISDC Data Centre for Astrophysics, University of Geneva, Ch. d'Ecogia 16, 1290 Versoix, Switzerland

53 IUCAA, Post Bag 4, Ganeshkhind, Pune University Campus, 411007 Pune, India

54 Imperial College London, Astrophysics group, Blackett Laboratory, Prince Consort Road, London, SW7 2AZ, UK

55 Infrared Processing and Analysis Center, California Institute of Technology, Pasadena, CA 91125, USA

56 Institut d'Astrophysique Spatiale, CNRS (UMR 8617) Université Paris-Sud 11, Bâtiment 121, 91405 Orsay, France

57 Institut d'Astrophysique de Paris, CNRS (UMR 7095), 98bis boulevard Arago, 75014 Paris, France

58 Institute for Space Sciences, 077125 Bucharest-Magurale, Romania

59 Institute of Astronomy and Astrophysics, Academia Sinica, 10617 Taipei, Taiwan

60 Institute of Astronomy, University of Cambridge, Madingley Road, Cambridge CB3 0HA, UK

61 Institute of Theoretical Astrophysics, University of Oslo, Blindern, 0315 Oslo, Norway

62 Instituto de Astrofísica de Canarias, C/Vía Láctea s/n, 38205 La Laguna, Tenerife, Spain

63 Instituto de Física de Cantabria (CSIC-Universidad de Cantabria), Avda. de los Castros s/n, 39065 Santander, Spain

64 Jet Propulsion Laboratory, California Institute of Technology, 4800 Oak Grove Drive, Pasadena, California, USA 
65 Jodrell Bank Centre for Astrophysics, Alan Turing Building, School of Physics and Astronomy, The University of Manchester, Oxford Road, Manchester, M13 9PL, UK

66 Kavli Institute for Cosmology Cambridge, Madingley Road, Cambridge, CB3 0HA, UK

67 LAL, Université Paris-Sud, CNRS/IN2P3, Orsay, France

68 LERMA, CNRS, Observatoire de Paris, 61 Avenue de l'Observatoire, 75014 Paris, France

69 Laboratoire AIM, IRFU/Service d'Astrophysique - CEA/DSM CNRS - Université Paris Diderot, Bât. 709, CEA-Saclay, 91191 Gifsur-Yvette Cedex, France

70 Laboratoire Traitement et Communication de l'Information, CNRS (UMR 5141) and Télécom ParisTech, 46 rue Barrault, 75634 Paris Cedex 13, France

71 Laboratoire de Physique Subatomique et de Cosmologie, Université Joseph Fourier Grenoble I, CNRS/IN2P3, Institut National Polytechnique de Grenoble, 53 rue des Martyrs, 38026 Grenoble Cedex, France

72 Laboratoire de Physique Théorique, Université Paris-Sud 11 \& CNRS, Bâtiment 210, 91405 Orsay, France

73 Lawrence Berkeley National Laboratory, Berkeley, California, USA

74 Max-Planck-Institut für Astrophysik, Karl-Schwarzschild-Str. 1, 85741 Garching, Germany

75 McGill Physics, Ernest Rutherford Physics Building, McGill University, 3600 rue University, Montréal, QC, H3A 2T8, Canada

76 Niels Bohr Institute, Blegdamsvej 17, 2100 Copenhagen, Denmark
77 Observational Cosmology, Mail Stop 367-17, California Institute of Technology, Pasadena, CA, 91125, USA

78 Optical Science Laboratory, University College London, Gower Street, London, UK

79 SISSA, Astrophysics Sector, via Bonomea 265, 34136, Trieste, Italy

80 School of Physics and Astronomy, Cardiff University, Queens Buildings, The Parade, Cardiff, CF24 3AA, UK

81 School of Physics and Astronomy, University of Nottingham, Nottingham NG7 2RD, UK

82 Space Research Institute (IKI), Russian Academy of Sciences, Profsoyuznaya Str, 84/32, 117997 Moscow, Russia

83 Space Sciences Laboratory, University of California, Berkeley, California, USA

84 Stanford University, Dept of Physics, Varian Physics Bldg, 382 via Pueblo Mall, Stanford, California, USA

85 Sub-Department of Astrophysics, University of Oxford, Keble Road, Oxford OX1 3RH, UK

86 UPMC Univ. Paris 06, UMR7095, 98bis boulevard Arago, 75014 Paris, France

87 Université de Toulouse, UPS-OMP, IRAP, 31028 Toulouse Cedex 4, France

88 Universities Space Research Association, Stratospheric Observatory for Infrared Astronomy, MS 232-11, Moffett Field, CA 94035, USA

89 Warsaw University Observatory, Aleje Ujazdowskie 4, 00-478 Warszawa, Poland 